\title{
« La toute-puissance de la barbe » Jenny P. d'Héricourt et les novateurs modernes
}

\section{Caroline ARNI}

\section{(2) OpenEdition}

1 Journals

\section{Édition électronique}

URL : https://journals.openedition.org/clio/139

DOI : $10.4000 /$ clio. 139

ISSN : 1777-5299

Éditeur

Belin

Édition imprimée

Date de publication : 1 avril 2001

Pagination : 145-154

ISBN : 2-85816-577-7

ISSN : 1252-7017

Référence électronique

Caroline ARNI, « «La toute-puissance de la barbe » Jenny P. d'Héricourt et les novateurs modernes », Clio. Histoire, femmes et sociétés [En ligne], 13 | 2001, mis en ligne le 19 juin 2006, consulté le 23 avril 2022. URL : http://journals.openedition.org/clio/139 ; DOI : https://doi.org/10.4000/clio.139

Ce document a été généré automatiquement le 23 avril 2022.

Tous droits réservés 


\title{
«La toute-puissance de la barbe » Jenny P. d'Héricourt et les novateurs modernes
}

\author{
Caroline ARNI
}

1 C'est un cercle extravagant mais tout à fait illustre - et pas du tout fortuit - qui est réuni dans les pages d'un livre en 1860: d'abord messieurs Pierre Leroux, Emile de Girardin (plutôt brusque), Ernest Legouvé (un sourire sur les lèvres), Jules Michelet (les larmes aux yeux), Auguste Comte (venant d'outre-tombe) et Pierre-Joseph Proudhon, ensuite un communiste anonyme, un philadelphe, un icarien, un Saint-Simonien orthodoxe, un fusionien et un phalanstérien. Et puis il y a encore un « moi » qui raconte l'événement : Jenny P. d'Héricourt. La rencontre des «novateurs modernes », ainsi que le suggère le récit, s'est faite à l'initiative de cette dernière, et c'est également elle qui invite ces messieurs présents à comparer leurs opinions « devant le public votre juge ». Finalement, Jenny d'Héricourt se charge également du recensement des voix de "l'honorable assemblée ». Le sujet dont il est question, c'est la liberté de la femme et l'égalité des sexes, et le résultat du vote est sans équivoque : «l'immense majorité de ceux qui pensent sont, à différents degrés, pour notre Emancipation ». Seuls MM. Comte, Proudhon et Michelet se prononcent contre l'affranchissement de la femme. Mais pour d'Héricourt, il n'y a aucune raison de classer l'affaire : « Maintenant que mes lecteurs sont au fait de vos opinions diverses, Messieurs, à moi, femme, de parler, de moi-même pour mon droit, sans m'appuyer sur autre chose que sur la Justice et la Raison $»^{1}$.

2 Evidemment, ce débat entre Académiciens, hommes politiques, intellectuels et socialistes utopistes n'a jamais eu lieu sous la forme décrite par l'auteure. Il s'agit d'un récit fictif qui conclut le premier tome de l'œuvre La femme affranchie. Réponse à MM. Michelet, Proudhon, E. de Girardin, A. Comte et aux autres novateurs modernes (Bruxelles/Paris 1860) dont l'auteure est Jenny P. d'Héricourt. Dans sa mise en scène, le débat s'organise comme dans une société savante : c'est une manifestation publique et il y a un recensement des voix. Cependant, les paramètres d'un débat éclairé ne sont 
pas toujours respectés : lorsqu'un Michelet ou un Proudhon - l'un " doux comme miel » l'autre " amer comme absinthe $"^{2}$ - sont guidés par un chauvinisme masculin, lorsqu'un Comte plaide pour l'instauration d'une stricte hiérarchie de classe et de sexe, l'auteure voit l'effet ni de la justice et ni de la raison.

Instaurer une société juste et raisonnable, voilà le combat de d'Héricourt. «Élève des doctrines résumées par notre glorieuse Révolution $»^{3}$, elle défend une théorie du social inspirée des Lumières et de la philosophie critique de Kant. Le débat fictif de d'Héricourt ne traite pas uniquement de la question de la liberté des femmes. Plus encore, ce discours porte sur un problème qui préoccupe la pensée française depuis 1789 et qui se pose dans un contexte de profonds changements politiques, sociaux et économiques: à savoir la question de l'ordre social. D'emblée cette question a été intimement liée à celle de la position sociale de la femme, comme le souligne Geneviève Fraisse: «En parallèle à l'élaboration d'un nouveau lien social, un autre lien sexuel s'avère nécessaire ${ }^{4}$.

Itinéraire d'une fille du siècle, itinéraire d'une intellectuelle

Le salon de Mme Fauvety était l'un des lieux à Paris où l'on pouvait débattre de l'ordre social et de la question des femmes. S'y réunissait le réseau gravitant autour de la Revue philosophique et religieuse, publication proche de la "libre pensée ». D'après Juliette Adam, on y discutait des sujets culturels et philosophiques et d'Héricourt "avait acquis dans ce milieu une autorité justifiée par des sérieuses études " 5 . Ses critiques des doctrines contemporaines concernant la femme publiées dans la Revue provoquèrent en effet l'attention et l'admiration du public. "L'article fit sensation » rapporte l'un des collaborateurs de la revue au sujet d'une de ses publications, «les écrivains mâles de la Revue se regardèrent à la manière des augures, le public applaudit jusque par delà les mots!». Ce qui avait impressionné avant tout, c'était «la science entre les mains de madame d'Héricourt $»^{6}$. Pourtant, exclue en tant que femme de l'académie et des universités, d'Héricourt dut affronter de multiples résistances pour avoir accès au savoir. En véritable « fille de [son] siècle $»^{7}$, elle fit l'expérience dans sa propre chair de l'inégalité et entreprit de défendre son sexe.

Née en 1809 à Besançon sous le nom de Jeanne-Marie-Fabienne Poinsard, d'Héricourt grandit dans un milieu d'artisans, dominé par le protestantisme et les idées républicaines ; à partir de 1817, elle vit à Paris ${ }^{8}$. Après une formation d'enseignante, elle étudie en autodidacte et au moyen de cours privés l'anatomie, la physiologie et l'histoire naturelle, elle acquiert un diplôme en médecine homéopathique, suit une formation de sage-femme à la Maternité et travaille dans les années 1850 comme gynécologue et pédiatre.

6 Jenny P. d'Héricourt entame son engagement publiciste et politique dans les années 1840 avec deux romans de critique sociale ; elle le poursuit dans le mouvement utopiste

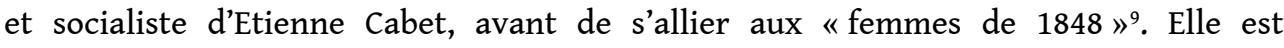
secrétaire de la Société pour l'Émancipation des femmes, il est possible qu'elle ait écrit également des articles sous le pseudonyme de Jeanne-Marie dans La Voix des femmes. Sa critique des "contre-émancipateurs $»^{10}$, à savoir Proudhon et Comte, et des doctrines chrétiennes qu'elle publie en 1850 dans la Revue philosophique et religieuse, est traduite en différentes langues européennes et a largement contribué au renouveau du débat européen sur la question de la femme. Son influence semble prouvée surtout en Russie et en Italie. 
7 D'Héricourt passe la décennie suivante aux États-Unis dans le cercle des mouvements féministes américains. Au début des années 1870, elle rentre à Paris, où elle meurt en 1875, appauvrie et seule. Le divorce étant interdit, d'Héricourt est restée mariée durant toute sa vie mais elle s'était séparée en fait de son mari en 1836, après quatre ans de mariage.

Machine à raisonnement ou : le sexe du discours public

8 Les amitiés informelles et privées ainsi que les débats publics de la Revue philosophique et religieuse étaient d'autant plus importants pour Jenny d'Héricourt que les féministes se trouvaient isolées dans la France de 1851 et que les femmes restaient toujours exclues de la politique et de l'académie. Comme publiciste, d'Héricourt réussit à faire entendre sa voix d'intellectuelle dans le débat contemporain à propos de l'ordre social et de la question de l'égalité des sexes. Elle dénonçait ainsi haut et fort l'exclusion des femmes du domaine public et de la politique. En contestant la « loi naturelle » autant par ses idées que par le fait même de son apparition sur scène, elle lançait un défi considérable à ses contemporains.

9 Pierre-Joseph Proudhon fut l'un de ceux qui répondirent à cette provocation. Dans sa réplique particulièrement grossière à l'un des articles critiques de décembre 1856, il fit savoir à d'Héricourt qu'en vue d'une éventuelle suite du débat, elle ferait mieux de se faire remplacer par un "parrain $»^{11}$. Non seulement, il craignait de violer les "bienséances " lors d'un débat violent, mais il se servait encore de tout son savoir anatomique et physiologique pour convaincre Mme d'Héricourt de l'impossibilité de ses actions : «Il y a chez vous, au cerveau comme dans le ventre, certain organe incapable par lui-même de vaincre son inertie native et que l'esprit mâle est seul capable de faire fonctionner, ce à quoi il ne réussit même pas toujours $»^{12}$. En se rapportant à l'infériorité intellectuelle du sexe féminin, Proudhon délimitait très nettement le territoire que Mme d'Héricourt tentait de quitter. En la renvoyant à sa condition de femme, il lui fit bien remarquer qu'elle n'était pas à sa place dans le domaine du débat public.

D'autres contemporains partageaient l'irritation de Proudhon, mais ils cherchèrent les explications ailleurs. Jules de Goncourt jugea Jenny d'Héricourt avec un respect ironique : "Elle a dans le style la toute-puissance de la barbe ${ }^{13}$. D'autres trouvèrent des termes plus malveillants pour exprimer leur indignation: selon eux, Jenny d'Héricourt était brutale, sans cœur, une «machine à raisonnement $»^{14}$. Cette perception comme "non-femme » est tout à fait révélatrice. En prenant la parole dans la polémique autour de la nature et du droit de la femme, d'Héricourt s'arrogeait une position de sujet dans le discours même qui la définissait comme objet en fonction de son sexe. Se comportant comme un homme, elle ne pouvait être une femme, ni même un être humain. Effectivement, son intervention ébranlait l'ordre des choses : ce que d'Héricourt mettait en question n'était pas uniquement des doctrines misogynes, mais le sexe masculin du discours théorique et politique. Pourtant, Jenny d'Héricourt voyait son statut d'intellectuelle menacé : l'on pouvait tout simplement, comme le faisait Proudhon, lui refuser le débat.

L'annihilation sociale de la femme

11 Malgré ces obstacles, d'Héricourt continua à écrire dans la Revue philosophique et religieuse et plus tard dans La femme affranchie contre ce qu'elle appelait "l'annihilation sociale de la femme " ${ }^{15}$, à savoir l'exclusion de la femme du travail et de la politique, de la citoyenneté et du droit à l'autonomie et à l'individualité. Reprenant à 
son tour les arguments scientifiques, elle contestait par exemple le fait que la biologie détermine l'existence sociale et en particulier l'infériorité de la femme: «l'Anatomie vous dit : chez les deux sexes, la masse cérébrale est semblable pour la composition et, ajoute la Phrénologie, pour le nombre des organes ${ }^{16}$. Il s'en suit que la distinction entre des hommes autonomes et des femmes assujetties n'est pas une loi naturelle, mais une décision politique et une pratique sociale : «Eh! non, Messieurs, ce ne sont pas là des hommes et des femmes: ce sont les tristes produits de votre égoïsme, de votre affreux esprits de domination, de votre imbécillité... ${ }^{17}$. D’Héricourt ne conteste pas qu'une " classification des sexes " puisse exister au delà de l'influence sociale, mais à son avis, celle-ci ne peut pas être reconnue ou théorisée dans une société imprégnée par une idéologie misogyne qui proclame comme « fait naturel » ce qui est, en fait, une pathologie sociale reproduite par les mœurs et l'éducation et réifiée par des théories insensée ${ }^{18}$. Par conséquent, chaque théorie de la différence des sexes ne pouvait être qu'idéologie pure et engendrait l'inégalité sociale. En deçà des essentialismes et biologismes, d'Héricourt faisait des relations entre les sexes non pas seulement une affaire politique mais aussi un objet de l'analyse critique du social.

Certes, d'Héricourt répondit vigoureusement à Proudhon: «M. Proudhon, reculant devant la discussion, ne peut échapper à ma critique $»^{19}$. Mais il n'en demeure pas moins que la précarité de son statut d'intellectuelle a perduré dans l'historiographie.

L'annihilation historiographique d'une théoricienne

La question de savoir comment est possible un lien social stable dans la société moderne est considérée comme la problématique constitutive de la sociologie. Ainsi, lorsqu'on analyse le discours social et théorique du xix ${ }^{e}$ siècle dans toute son envergure comme précurseur de la sociologie moderne, il s'ensuit qu'un Comte devient le " père fondateur " et qu'un Proudhon est lui aussi classé parmi les sociologues. On cherchera en vain le nom de Jenny d'Héricourt dans cette pré-histoire de la sociologie bien que sa " théorie philosophique du droit » puisse être lue comme une théorie du social ${ }^{20}$.

En fait, il s'agit pour d'Héricourt de poser la question de l'ordre social en évitant les réductionnismes de la pensée utilitariste comme ceux de la théorie collectiviste de Comte, car « s'il est mauvais que le collectif soit absorbé par l'individuel, il ne l'est pas moins que l'individu soit absorbé par le collectif $»^{21}$. À partir de cette problématique, elle développe les fondements d'un concept de l'ordre social qu'Émile Durkheim, élève de son ami et collaborateur Charles Renouvier, reprendra vers la fin du XIX siècle sous le nom de "solidarité organique " et qui deviendra l'un des piliers de la tradition sociologique ${ }^{22}$. Il s'agit en effet de l'idée que l'individualisation dans une société basée sur la division du travail ne doit pas forcément aboutir à la désintégration, mais qu'elle rend possible l'intégration en fondant la coopération. Se faisant, d'Héricourt comprend les hommes et les femmes dans son analyse - à la différence de ce que fera Durkheim. Selon elle, des femmes participent également au "progrès des lumières ", ce qui veut dire qu'elles participent au processus de modernisation qui libère l'individu de ses attaches traditionnelles à la famille et à la reproduction, qui fait s'épanouir son individualité et le transforme en une "force sociale»" Par conséquent, l'affranchissement de la femme n'est pas seulement un devoir de la justice, mais tout autant une nécessité sociale : si la solidarité est fondée par le besoin réciproque des humains ayant des facultés très diverses, alors la cohésion sociale n'est possible que par le biais de l'épanouissement libre de tous les individus, y compris les femmes.

Une intellectuelle, un rêve 

l'ordre social au détriment de l'individualité féminine. Et c'est justement cet enjeu de l'affranchissement des femmes qui lui a permis de développer une pensée théorique du social qui conçoit la société moderne en tant que «collection organisée des individus qui la composent $»^{24}$. Cependant, parce qu'elle a développé sa pensée théorique sous le signe du «lien sexuel » égalitariste, on la considère exclusivement comme une théoricienne de la pensée féministe et on ne l'inclut pas dans les théories sociologiques. Alors qu'au XIx ${ }^{e}$ siècle la relation étroite entre «lien social » et «lien sexuel » était évidente aux penseurs (principalement au détriment de l'individualité féminine), elle est méconnue par les historiens de la pensée sociologique. C'est ainsi autant la dimension sexuée du discours sociologique qui est ignorée que la dimension sociologique du discours féministe. dans ces quelques pages de La femme affranchie n'est ni pure fiction ni simple illustration d'une polémique. Plus qu'une analyse ironique et précise d'une discussion intellectuelle et politique de son temps, cette mise en scène permet l'affirmation de soi d'une femme qui initie des discussions, qui commente, qui analyse et qui critique ; une femme donc qui revendique le statut d'intellectuelle parmi des intellectuels. Moyennant une fiction, d'Héricourt s'inscrit dans un discours qui ne lui accorde qu'une participation fragile et dont elle sera presque entièrement exclue rétrospectivement. En ce sens, ce débat fictif est peut-être la mise en scène d'un rêve: le rêve qu'une intellectuelle puisse transformer les pratiques sociales et politiques.

Traduit par Ursula Kaeser

\section{BIBLIOGRAPHIE}

Adam Juliette, 1904, Mes premières armes littéraires et politiques, Paris, Lemerre.

Albistur Maïté et Armogathe Daniel, 1977, Histoire du féminisme français, Paris, Des Femmes.

Anteghini Alessandra, 1988, Socialismo e femminismo nella Francia del XIX secolo : Jenny d'Héricourt, Genova, ECIG.

Arni Caroline et Honegger Claudia, 1998, « Jenny P. d'Héricourt (1809-1875). Weibliche Modernität und die Prinzipien von 1789 », Honegger Claudia / Wobbe Theresa, 1998, Frauen in der Soziologie. Neun Porträts, München, Beck Verlag, pp. 60-98.

Arni Caroline, 1998, femme sociologue - femme diable. Jenny P. d'Héricourt - eine frühsoziologische Denkerin im Frankreich des 19. Jahrhunderts, Bern, Schriftenreihe Kultursoziologie des Instituts für Soziologie.

--, 1998, « Erstrittene Öffentlichkeit. Jenny P. d'Héricourts Auseinandersetzung mit den Theorien von Geschlecht und Gesellschaft », in Brigitte Studer, Regina Wecker, Béatrice Ziegler (dir.), Frauen und Staat / Les Femmes et l'État, Basel, ITINERA 20, pp. 77-89. 
Chouippe A., 1857, « Revue critique de la Revue philosophique et religieuse », La Revue philosophique et religieuse, VI (janvier), pp. $131 \mathrm{ff}$.

D’héricourt Jenny P., 1855, « Le catéchisme positiviste de M. Auguste Comte », La Revue philosophique et religieuse, III (décembre), pp. 47-61.

,-- 1856 , « M. Proudhon et la question des femmes », La Revue philosophique et religieuse, VI (décembre), pp. 5-15.

--, 1857a, « Réponse de Mme Jenny d'Héricourt à M. P. J. Proudhon », La Revue philosophique et religieuse, VI (février), pp. 267-275.

,$-- 1857 \mathrm{~b}$, « M. Proudhon et la question des femmes », La Revue philosophique et religieuse, VI (mars), pp. 521-522.

--, 1860, La femme affranchie. Réponse à MM. Michelet, Proudhon, E. de Girardin, A. Comte et aux autres novateurs modernes, 2 volumes, Bruxelles/Paris, Lacroix, Van Meenen et Cie. (et al.).

Durkheim Émile, 1996 [1993], De la division du travail social, Paris, Presses Universitaires de France. Fauré Christine, 1991, « Weibliche Intellektuelle und Staatsbürgerschaft. Frankreich von der Revolution bis zum Zweiten Kaiserreich (1789-1870) », L'Homme. Zeitschrift für Feministische Geschichtswissenschaft, 2, pp. 33-47.

Fraisse Geneviève, 1992, La Raison des femmes, Paris, Plon.

Offen Karen, 1987, « Qui est Jenny P. d'Héricourt ? Une identité retrouvée », Bulletin de la Société d'Historier de la Révolution de 1848 et des révolutions du XIXe siècle, III, pp. 87-100.

Proudhon Pierre-Joseph, 1857, « Lettre à Madame J. d'Héricourt », La Revue philosophique et religieuse, VI (janvier), pp. 164-166.

Riot-Sarcey Michèle, 1994, La démocratie à l'épreuve des femmes. Trois figures critiques du pouvoir 1830-1848, Paris, Albin Michel.

Rochefort Florence, 2000, «Féminisme et protestantisme au XIX ${ }^{\mathrm{e}}$ siècle, premières rencontres 1830-1900 », Bulletin de la Société de l'histoire du protestantisme français, t. 146, pp. 69-89.

\section{NOTES}

1.D’Héricourt 1860, t. I : 221, 228, 229.

2.D'Héricourt 1860, t. I : 94.

3.D’Héricourt 1860, t. II : 7.

4.Fraisse $1992: 49$.

5.Adam $1904: 31$.

6.Chouippe $1857: 147 f$.

7.D’Héricourt 1860, t. I : 7.

8.Pour la biographie de d'Héricourt cf. Offen 1987, Anteghini 1988. Christine Fauré dénonce à juste titre le manque d'informations sur la biographie de d'Héricourt (Fauré 1991). Elle souligne en même temps que l'approche purement biographique de l'histoire de la pensée féministe contient des leurres. Peut-être que le manque d'information biographique s'avère finalement être un avantage, dans la mesure où cela favorise une analyse intense du matériel publié que des femmes comme Jenny d'Héricourt ont laissé. Il me semble en effet qu'aussi bien son œuvre La femme affranchie que ces différents 
articles dans la Revue philosophique et religieuse mériteraient plus d'analyses. Pour d'Héricourt dans le contexte du féminisme protestante cf. Rochefort 2000.

9.Riot-Sarcey 1994 : 187, 322, 325. D'Héricourt s'est détourné de Cabet, parce que celuici n'accordait pas de position politique autonome à la question de la femme. En se détournant de lui, d'Héricourt n'a pourtant pas abandonné les idées socialistes et la solidarité avec la classe ouvrière. Dans plusieurs passages de La femme affranchie elle met en parallèle la naissance de l'inégalité sociale par la « classification de l'espèce humaine soit en castes, en classes, en sexe. » (D'Héricourt 1860, t. II : 123). Dans son court débat avec Proudhon, elle souligne à plusieurs reprises qu'elle regrette de devoir le critiquer aussi violemment, lui, dont elle approuve et respecte les idées.

10.D'Héricourt 1860, t. I : 8.

11.Cf. d'Héricourt 1856, Proudhon janvier 1857.

12.Proudhon $1857: 167,165$.

13.Jules de Goncourt cit. d'après Adam 1904 : 55.

14.Cit. d'après d'Héricourt 1860, t. I : 10

15.D'Héricourt 1860, t. I : 166.

16.D'Héricourt 1860, t. I : 178.

17.D'Héricourt 1860, t. II : 124.

18.D'Héricourt 1860, t. II : 122.

19.D'Héricourt 1857b : 521.

20.Cf. Arni 1998, Arni/Honegger 1998.

21.D'Héricourt $1855: 57$.

22.Durkheim [1893] 1996. Je n'ai pas encore retracé la lignée d'Héricourt - Renouvier Durkheim de manière systématique. Il n'est toutefois pas exclu que Durkheim a pu connaître les écrits de d'Héricourt.

23.D'Héricourt 1860, t. I : 7.

24.D'Héricourt 1860, t. I : 42f.

\section{RÉSUMÉS}

Quand en 1856 Jenny P. d'Héricourt (1809-1875), sage-femme, féministe et philosophe, critiqua Pierre-Joseph Proudhon pour sa théorie sur l'infériorité féminine, celui-ci refusa tout discussion, invoquant son infériorité intellectuelle naturelle. Néanmoins d'Héricourt continua de publier de ferventes critiques des théories des philosophes sociaux de son époque sur l'inégalité des sexes. L'article veut d'une part éclairer la notion d'intellectuelle et les conditions nécessaires pour agir comme telle. D'autre part, il questionne les limites d'une existence intellectuelle féminine telles qu'elles se présentent non seulement dans le refus des contemporains mais aussi dans l'historiographie de la pensée sociologique qui ignore la contribution d'Héricourt aux questions fondatrices du discours sociologique anticipant un concept clé d'Émile Durkheim.

When the French midwife, feminist activist and social philosopher Jenny P. d'Héricourt (1809-1875) criticised Pierre-Joseph Proudhon in 1856 for his theory on female inferiority, he invoked her innate lack of intellectual ability to refuse all public discussion with her. Nonetheless d'Héricourt continued to publicly criticise the misogynist elements and the theoretical defects in 
the work of contemporary social philosophers. This article sheds light on d'Héricourt's selfunderstanding as an intellectual and on the conditions necessary for women to act as such. At the same time it points out the boundaries of this female intellectuality, impose not only by her contemporaries but also by the historiography of social thought which ignores d'Héricourt as a social philosopher who anticipated a theoretical framework later made famous by Émile Durkheim.

\section{AUTEUR}

\section{CAROLINE ARNI}

Caroline Arni est assistante à l'institut de sociologie de l'Université de Berne. Travaille sur le mariage et les relations intimes des sexes à l'époque contemporaine, histoire et sociologie des sciences humaines (spécialement l'aspect du genre), histoire et sociologie de la pensée féministe. A rédigé son mémoire de sociologie sur Jenny d'Héricourt (- femme sociologue - femme diable. Jenny P. d'Héricourt : Eine frühsoziologische Denkerin im Frankreich des 19. Jahrhunderts, Schriftenreihe des Instituts für Soziologie, Bern 1998) et a publié divers articles. Sa dernière publication porte sur « Robert Grimms Befreiungsschlag. Eine Fallstudie zum Verhältnis von Geschlechtergeschichte und politischer Geschichte der Schweiz », Traverse, 7, 1, 2000, pp. 109-124. 